\title{
Syndrome de Klinefelter et microinjections intraovocytaires de spermatozoïdes (revue de la littérature)
}

\author{
Gérald RAVEROT, Hervé LEJEUNE \\ Département de Médecine de la Reproduction, Hôpital E. Herriot et INSERM-INRA U418, \\ Hôpital Debrousse, Lyon
}

RÉSUMÉ

Le syndrome de Klinefelter représente historiquement la forme clinique typique de l'azoospermie sécrétoire sans espoir d'obtenir une paternité biologique. Les méthodes de microinjection intraovocytaire d'un spermatozoïde, soit de l'éjaculât, soit extrait du parenchyme testiculaire, ont pu être appliquées à des patients présentant un syndrome de Klinefelter. Les publications que nous avons recensées jusqu'en juin 2002 font état de microinjections, soit à partir de spermatozoïdes éjaculés, dans 9 cas de syndrome de Klinefelter avec oligospermie extrême, avec pour résultats globaux : 79 ovocytes matures, 52 ovocytes fécondés, 31 embryons, 18 embryons transférés, 6 grossesses, 5 naissances, soit à partir de spermatozoïdes testiculaires, dans 93 cas, avec pour résultats globaux : 347 ovocytes, 193 ovocytes fécondés, 149 embryons, 78 embryons transférés sur 37 cycles, 24 grossesses cliniques et 2 grossesses biochimiques, et 32 naissances. Bien que l'on puisse tout à fait suspecter un biais de publication (les tentatives ayant donné lieu à des grossesses ayant été publiées, les échecs n'ayant vraisemblablement pas fait l'objet de publication), ces résultats sont tout de même inattendus si on se réfère au pronostic traditionnellement admis dans le syndrome de Klinefelter. Le taux de spermatozoïdes aneuploïdes semble lui aussi inférieur à ce que l'on pouvait craindre. La proportion réelle des cas de syndrome de Klinefelter pour lesquels on peut disposer de spermatozoïdes ayant un bon potentiel de développement embryonnaire reste toutefois à établir et les moyens permettant de repérer ces patients restent à préciser.
Mots Clés : Klinefelter, azoospermie, extraction, spermatozoïde, testicule, microinjection, biopsie

\section{INTRODUCTION}

L'incidence du syndrome de Klinefelter est de 1/600 nouveau-nés mâles [26] mais atteint près de 3,1\% dans les populations d'hommes infertiles [15]. Le syndrome de Klinefelter est généralement caractérisé par un hypogonadisme hypergonadotrophique avec atrophie testiculaire majeure, azoospermie et gynécomastie. Le diagnostic peut être fait lors du bilan d'un retard pubertaire ou bien souvent lors d'un bilan d'infertilité. La cause de cette dysgénésie est une aneuploïdie chromosomique avec une formule caryotypique $47, X X Y$. Dans $10 \%$ des cas une mosaïque $46, \mathrm{XY} / 47, \mathrm{XXY}$ est notée sur le caryotype, la présentation clinique est alors habituellement moins sévère avec une possible oligospermie $[23,30]$, des grossesses spontanées ont même été décrites [21,38]. Des îlots de spermatogenèse conservés et même des oligozoospermies sévères ont également été retrouvées chez des sujets $47, \mathrm{XXY}$ en dehors de toute mosaïque [41].

Les récentes avancées en matière d'aide médicale à la procréation (ICSI et TESE) ont permis la prise en charge de ces patients 47,XXY par certaines équipes, et d'obtenir des grossesses à partir du sperme éjaculé $[2,4,7,17-19]$

\footnotetext{
Correspondance :

Pr. Hervé LEJEUNE - Département de Médecine de la Reproduction, Faculté de Médecine, Domaine Rockefeller, 8 avenue Rockefeller, 69008 Lyon Cedex 8 -

Tel 04.78.77.70.65 - Fax 04.78.77.72.64 -

Email lejeune@univ-Lyon1.fr
} 
ou à partir de biopsies testiculaires $[1,14,19,20,22,27$ $29,31-35,37,41-43]$.

L'objectif de cette revue est de faire la synthèse des résultats publiés jusqu'en juin 2002 en terme de nombre de tentatives (ayant donné lieu à publication), de nombre de grossesses et de naissances obtenues et d'évaluer le risque de transmission d'anomalies chromosomiques.

\section{FÉCONDATION IN VITRO AVEC MICRO- INJECTION DE SPERMATOZOÏDES ÉJACULÉS PAR DES SUJETS 47,XXY (Tableau 1)}

La première tentative fut publiée par Harari et al. en 1995 [17]. Cet article décrivait les premières tentatives de fécondation in vitro avec microinjection à partir de spermatozoïdes éjaculés d'un patient porteur d'un syndrome de Klinefelter (mosaïque avec $2 \%$ de cellules $46, \mathrm{XY}$ ) ayant permis d'obtenir une fécondation de tous les ovocytes murs injectés et le transfert de deux embryons mais sans grossesse. Depuis seules 6 études ont été publiées $[2,4,7,18,19$, 33]. L'ensemble de ces études comprend 9 patients présentant un syndrome de Klinefelter (dont 2 mosaïques), avec pour tous une oligospermie extrême. Sur les 10 cycles de FIV publiés que l'on peut analyser, on s'aperçoit que la fécondance est satisfaisante puisque 52 sur 79 ovocytes microinjectés $(65,8 \%)$ sont fécondés. Seule l'étude de Ron-El [33] est discordante mais dans cette étude les ovocytes ont été microinjectés avec des spermatozoïdes immobiles. Ces résultats font donc chuter le pourcentage de fécondance qui serait de 75,3\% (52/69) sans cette étude. De plus, 31 embryons seront obtenus à partir de ces 52 ovocytes fécondés $(59,6 \%)$.

Le pourcentage de grossesses est également satisfaisant puisque sur les 9 cycles ayant permis d'avoir 31 embryons, 6 grossesses ont été obtenues aboutissant à la naissance de 5 enfants ( 2 grossesses gémellaires [2, 4], une grossesse simple [7]), une grossesse biochimique et deux fausses couches spontanées. Rapporté au nombre d'embryons transférés, ce résultat est particulièrement bon puisque seuls 18 embryons ont été transférés, le taux d'implantation (nombre de sacs / nombre d'embryons transférés) est donc de $27,7 \%$.

Cinq embryons congelés n'ont pas été transférés et, dans l'étude de Bielenska et al. [2], un diagnostic préimplantatoire a été fait sur les 10 embryons obtenus et seuls les 3 embryons normaux ont été transférés (les autres embryons présentant des anomalies chromosomiques mais sans formule 47,XXY). Par ailleurs, l'analyse du matériel ramené par le curetage après la fausse couche spontanée dans l'étude de Hinney et al. [18] n'a pas révélé d'anomalie chromosomique.
Encouragés par ces résultats, et grâce au développement des techniques de microinjection de spermatozoïdes testiculaires (TESE-ICSI) chez des sujets présentant une azoospermie non obstructive [3, 8, 39], d'autres équipes ont étendu cette nouvelle option thérapeutique aux patients présentant un syndrome de Klinefelter.

\section{FÉCONDATION IN VITRO AVEC MICROINJECTION DE SPERMATOZOÏDES TESTICULAIRES DE SUJETS 47,XXY (Tableau 2)}

Les premières tentatives ont été rapportées par Tournaye et al. en 1996 [41], à propos de 9 patients 47,XXY non mosaïques dont seulement 3 présentaient une azoospermie, les 6 autres ayant une cryptozoospermie. Tous ont bénéficié d'une biopsie testiculaire et celle-ci a permis de retrouver des spermatozoïdes pour 4 d'entre eux. Trois patients ont bénéficié d'une FIV avec ICSI, à partir d'un total de 22 ovocytes microinjectés, 8 ont été fécondés permettant d'obtenir 6 embryons, 4 ont été transférés aboutissant à une grossesse biochimique. Dans cette première étude, des spermatozoïdes étaient retrouvés, après biopsie testiculaire, chez près de $50 \%$ des patients avec cependant une fécondance médiocre $(36 \%)$ mais donnant tout de même lieu à un développement embryonnaire.

De nombreuses autres études sont venues compléter celleci mais le nombre de patients reste limité. En effet, 17 articles ont été publiés concernant 93 patients présentant un syndrome de Klinefelter $[1,14,19,20,22,27-29,31-35$, $37,41-43$ ], l'age moyen était de 30,5 ans, le volume testiculaire était généralement faible, inférieur à $6 \mathrm{ml}$ sauf dans la série de Levron [22] où le volume était compris entre 7 et $10 \mathrm{ml}$. Tous ces patients avaient une formule carytotypique $47, X X Y$ homogène sauf un patient qui présentait une mosaïque $47, X X Y / 46, X Y$ [29]. Seuls 79 patients présentaient une azoospermie lors de la prise en charge, une cryptozoospermie était retrouvée pour 13 patients, enfin le dernier présentait une oligospermie extrême [19].

Tous ces patients ont donc bénéficié d'une biopsie testiculaire à la recherche de spermatozoïdes. Cette biopsie était réalisée soit par aspiration à l'aide d'aiguilles fines avec prélèvements multiples $[13,32-35,40,42]$ soit par large biopsie chirurgicale $[20,27,28,31,43]$.

Des spermatozoïdes mobiles ont pu être isolés à partir de ces biopsies chez 49 patients soit $52,7 \%$. Ces résultats sont comparables à ceux obtenus dans les séries de biopsies réalisées pour azoospermie non obstructives sans syndrome de Klinefelter : 63,9\% (série de 83 patients) pour Palermo et al. [29] ; 48\% (série de 179 patients) pour Tournaye et al. [42].

Les chances d'obtenir des spermatozoïdes mobiles sem- 


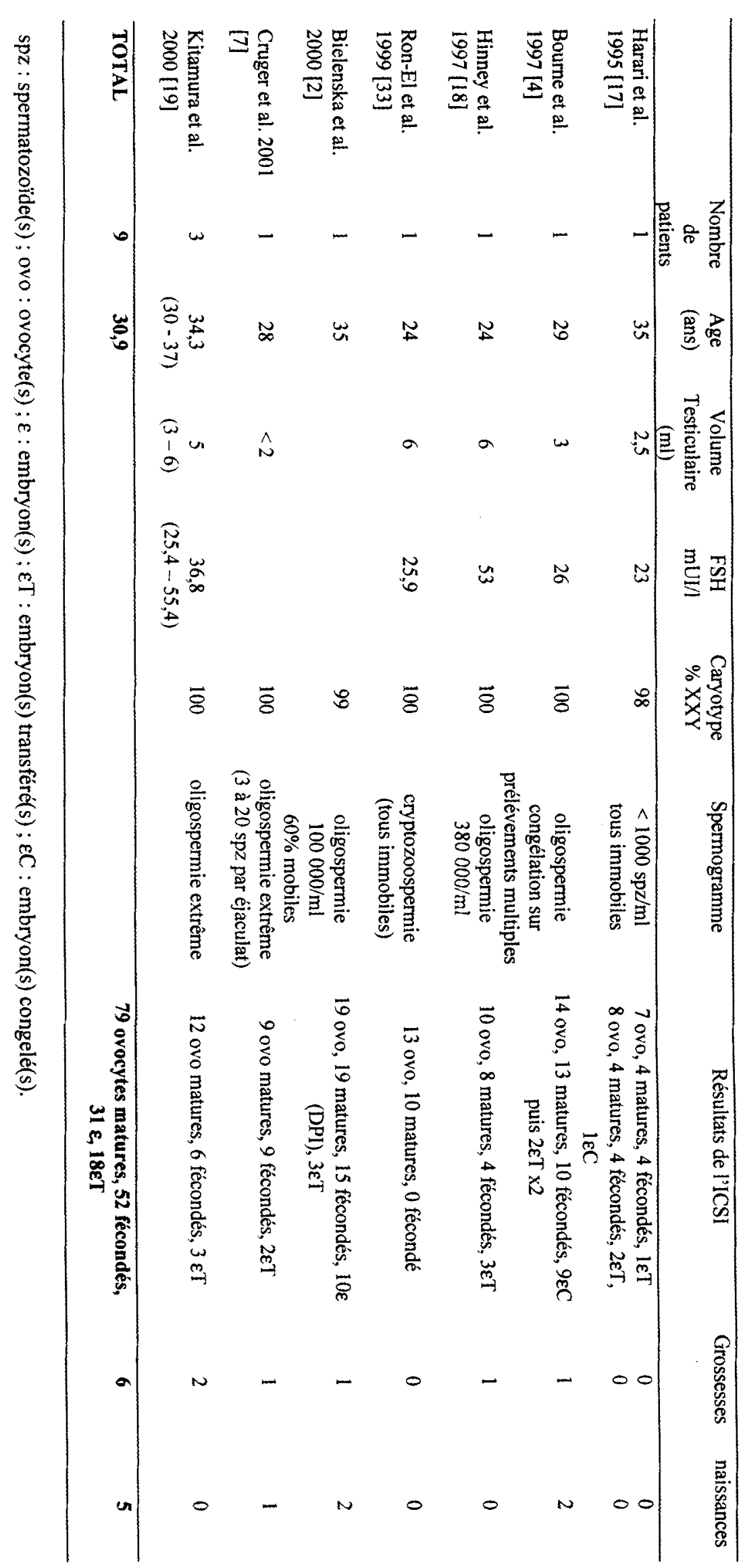




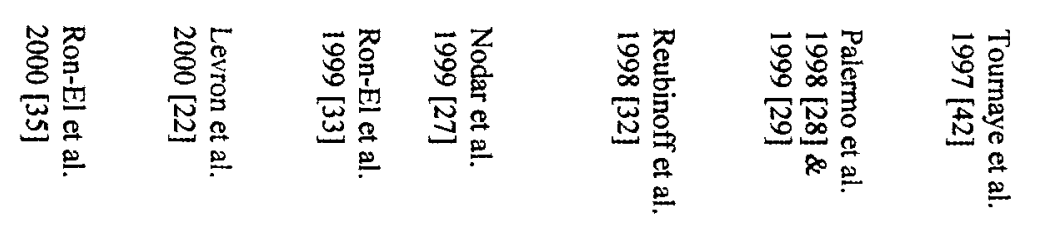

- - - -

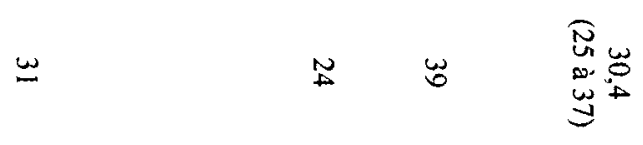

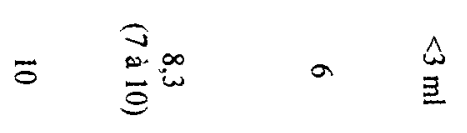

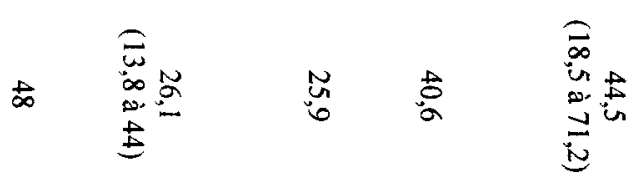

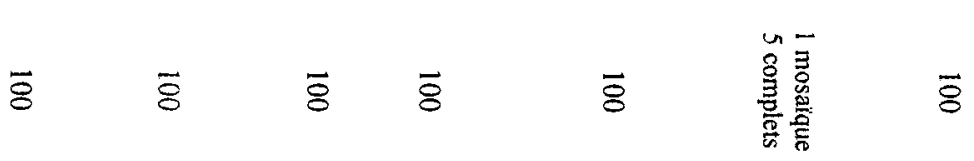

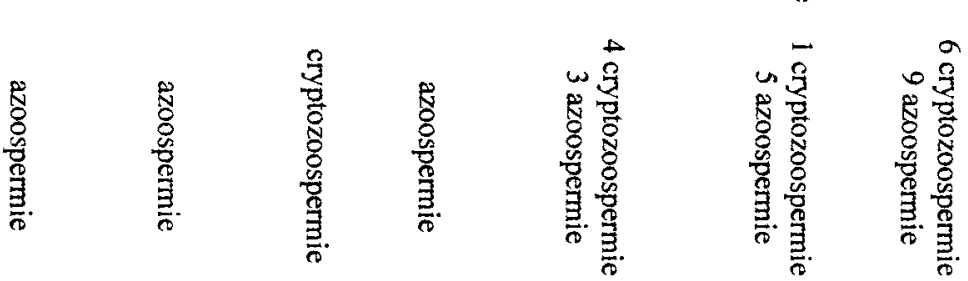

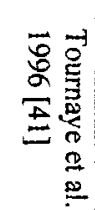

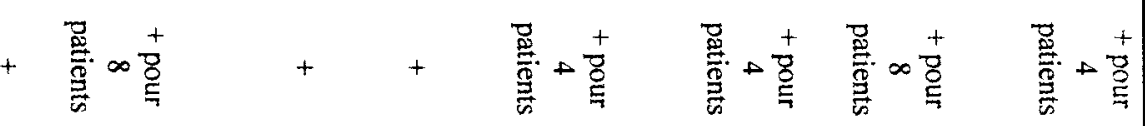

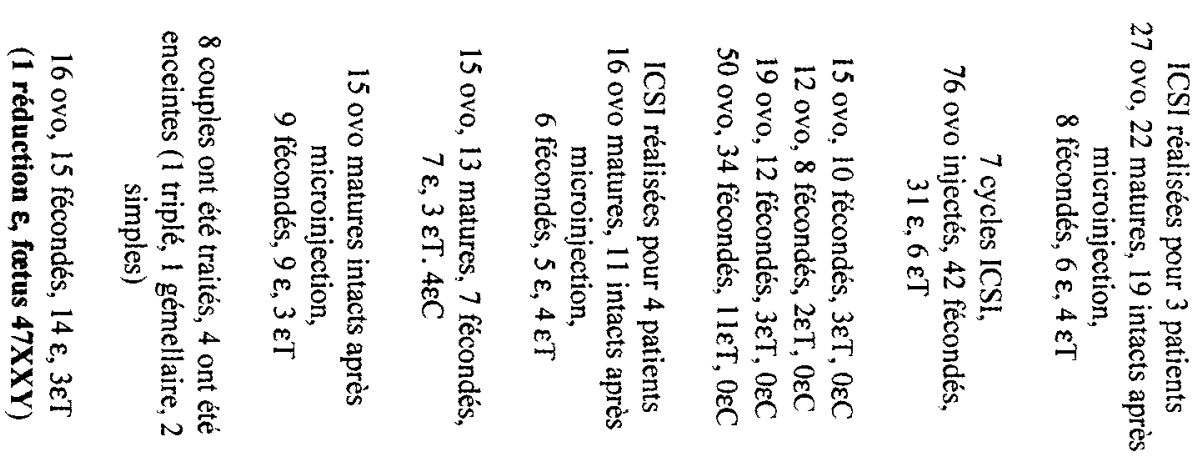

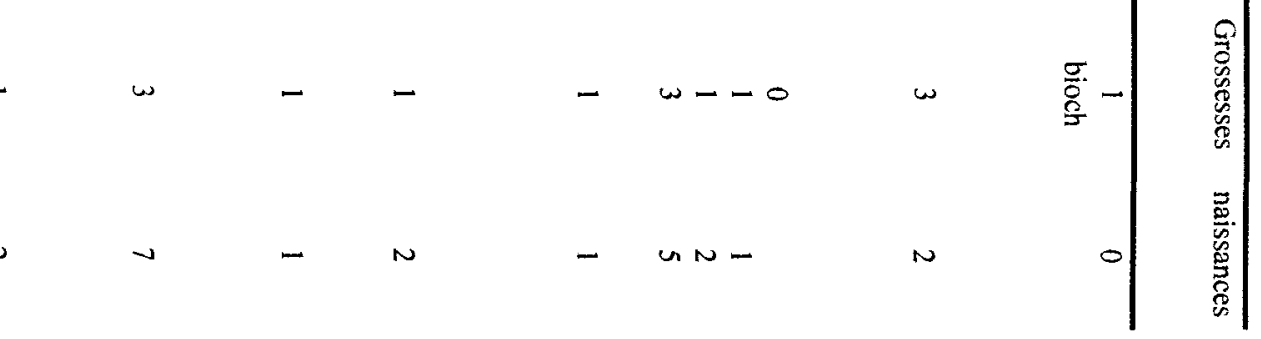

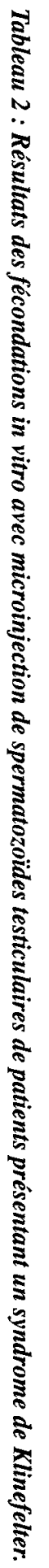




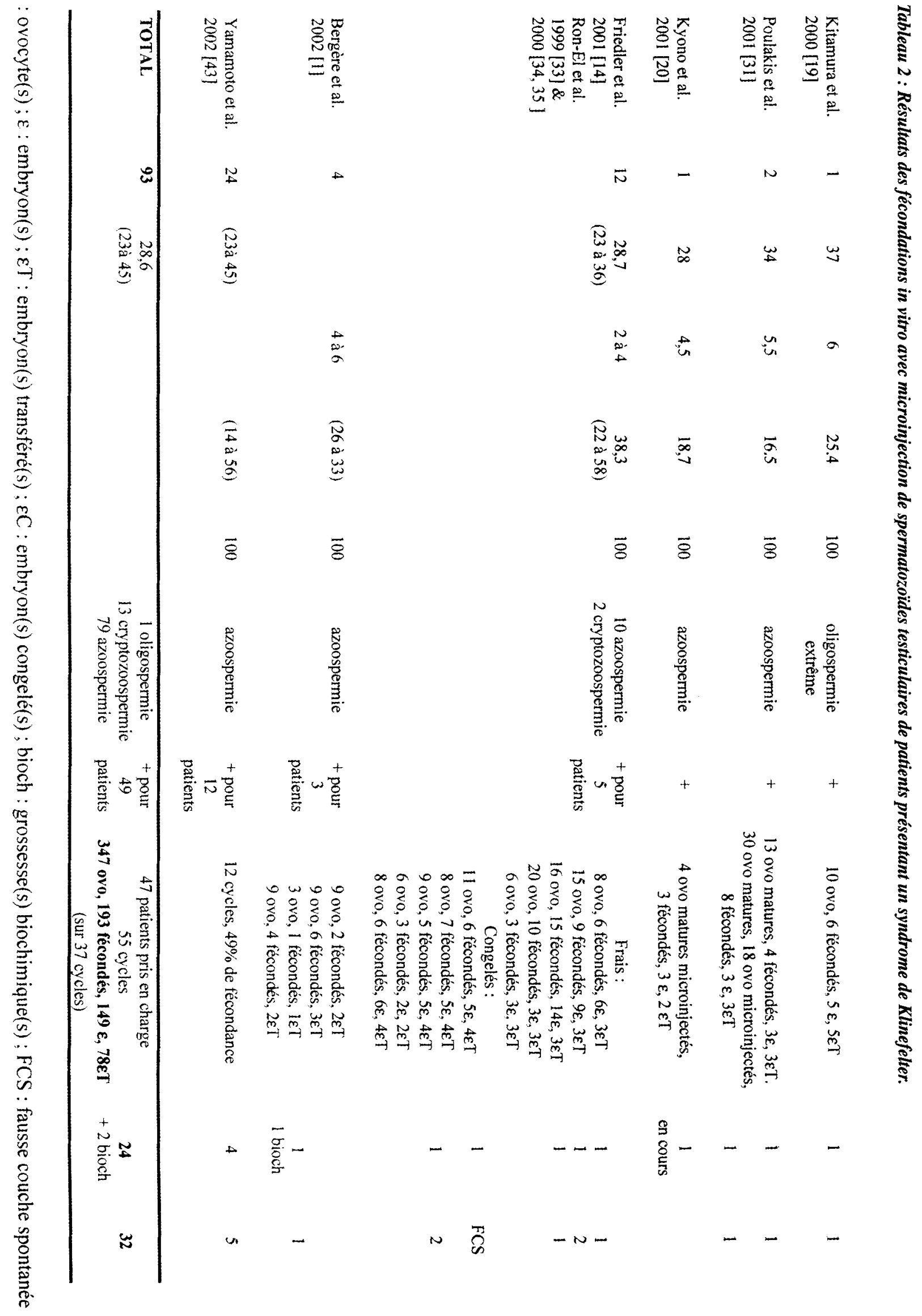


blent donc identiques aux autres causes d'azoospermie non obstructives. Il faut cependant nuancer ces résultats car ne sont probablement publiées que les séries dans lesquelles il a été trouvé des spermatozoïdes chez des patients présentant un syndrome de Klinefelter. Il existe donc vraisemblablement un biais de publication surestimant les chances d'obtenir des spermatozoïdes dans les biopsies en cas de syndrome de Klinefelter. Seules de grandes séries prospectives avec biopsie testiculaire systématique pourraient répondre précisément à cette question. On peut toutefois extrapoler que le pourcentage est proche de $50 \%$ puisqu'il est de $46,5 \%$ lorsque l'on n'étudie que les quatre plus grandes études publiées $[14,22,42,43]$ regroupant à elles quatre 71 patients (soit $76 \%$ des sujets étudiés).

Les résultats obtenus en terme de fécondation et de grossesse à partir de ces spermatozoïdes provenant des biopsies testiculaires sont également comparables à ceux de patients présentant une azoospermie non liée à un syndrome de Klinefelter. En effet, sur les 37 cycles que l'on peut analyser individuellement, le taux de fécondance est de 55,6\% (193 ovocytes fécondés / 347 ovocytes microinjectés) et globalement de $49 \%$ pour 12 couples dans l'étude de Yamamoto et al. [43] alors qu'il est de respectivement de 51 et $57 \%$ dans les études de Tournaye et al. [42] et de Palermo et al. [29] dans le cadre d'azoospermies non obstructives. De même le développement embryonnaire est satisfaisant, avec l'obtention de 82 embryons de bonne qualité $(42,4 \%)$, ainsi que l'implantation puisque 18 grossesses (dont 2 biochimiques) ont été obtenues à partir des 78 embryons transférés soit $23,1 \%$ d'implantation (ou $20,5 \%$ si l'on exclut les grossesses biochimiques).

Au total, 93 patients ont été explorés, la biopsie testiculaire à permis d'obtenir des spermatozoïdes chez 49 d'entre eux $(52,6 \%) ; 45$ sont entrés dans des cycles de FIV avec ICSI permettant d'obtenir 26 grossesses (dont 2 biochimiques) soit un pourcentage de grossesse de $55,3 \%$ (ou $53,3 \%$ si l'on exclut les grossesses biochimiques), et la naissance de 32 enfants sains ne présentant pas de malformation ni d'anomalie caryotypique.

Il faut là encore nuancer ces bons résultats par le biais de publications puisque depuis le premier article de Tournaye et al. [41], seules des tentatives ayant permis d'obtenir des grossesses ont été publiées. Il n'en reste pas moins que ces résultats sont meilleurs qu'attendus historiquement et que, dans les publications, les sujets présentant un syndrome de Klinefelter semblent avoir un pronostic proche de celui des sujets présentant une azoospermie non obstructive de cause inconnue.

\section{SYNDROME DE KLINEFELTER ET RISQUE DE TRANSMISSION}

Contrairement à ce qui se passe pour la majorité des azoospermies sécrétoires pour lesquelles l'étiologie et le devenir des enfants sont inconnus, dans le cas des syndromes de Klinefelter la cause est connue et le risque de transmission de l'anomalie chromosomique peut être exploré. Bien qu'aucun enfant, de père Klinefelter, ne soit né à ce jour avec un syndrome de Klinefelter, un caryotype réalisé sur des ponctions de villosités choriales au cours d'une décision de réduction embryonnaire dans le cadre d'une grossesse triple a révélé un fœus 47,XXY et deux autres fotus normaux $(46, X Y$ et $46, X X)$ [35]. La réduction a concerné le fotus $47, \mathrm{XXY}$ et le caryotype réalisé sur le liquide amniotique a confirmé le diagnostic. Le risque d'obtenir un embryon atteint d'un syndrome de Klinefelter est donc bien réel mais il reste difficile à évaluer. En effet, historiquement il était reconnu que seules les spermatogonies 46,XY étaient capables de terminer la méiose et d'aboutir à des spermatozoïdes normaux. Ainsi les spermatozoïdes obtenus proviendraient d'une mosaïque testiculaire et le risque de transmission à partir de spermatozoïdes obtenus chez des sujets présentant un syndrome de Klinefelter serait le même que dans la population générale. Cependant dès 1969, Skakkebaek [36] suggère que les cellules germinales $47, \mathrm{XXY}$ peuvent achever leur méiose et aboutir à la production de spermatozoïdes. Des études plus récentes ont confirmé ces observations en montrant une augmentation de l'incidence des cellules $24, X Y$ grâce à l'analyse caryotypique [6] ou par FISH [5, 9, 11, 16, 25] des spermatozoïdes éjaculés de patients présentant un syndrome de Klinefelter, mosaïque ou non. Ces différentes études montrent que plus de $90 \%$ des spermatozoïdes ont une formule chromosomique normale mais par contre que le nombre de spermatozoïdes $23, \mathrm{X}$ est significativement plus élevé que celui des $23, \mathrm{Y}$ avec un ratio de $2 / 1$ au lieu du 1/1 attendu. De plus, la fréquence des spermatozoïdes $24, X Y$ est significativement plus élevée avec des pourcentages allant de $0,92 \%$ [6] à 2,09\% [5] et même plus de $12,3 \%$ dans l'étude de Foresta et al. [11], sans augmentation des hypohaploïdies 22,0 attendues en cas de non disjonction du premier cycle de méiose suggérant donc la possibilité d'une méiose pour les spermatogonies 47,XXY. Ces résultats sont confortés par l'étude de Foresta et al. [12] qui ont analysé par FISH des prélèvements testiculaires obtenus par ponctions à l'aiguille fine chez des sujets présentant un syndrome de Klinefelter non mosaïque et qui retrouvent des spermatogonies et des spermatocyte I à caryotype $47, \mathrm{XXY}$ associés à des spermatides et des spermatozoïdes, ce qui suggère, d'après ces auteurs, la possibilité de méiose des cellules germinales $47, \mathrm{XXY}$ de ces patients.

Les conclusions de ces différentes études doivent toutefois être nuancées puisque Bergère et al. [1] ne retrouvent pas de déséquilibre dans le rapport $23, \mathrm{X} / 23, \mathrm{Y}$ et que le pourcentage d'aneuploïdie concernant les chromosomes $\mathrm{X}$ et $\mathrm{Y}$ 
n'est que de $2,7 \%$ contre $4 \%$ pour le chromosome 18 . Sans exclure la possibilité de méiose de cellules $47, \mathrm{XXY}$, l'hypothèse avancée est que la fréquence élevée d'aneuploïdie dans cette population correspond à des erreurs de méiose de spermatogonies normales situées dans un testiculaire défavorable comme cela a été décrit par Finkelstein et al. [10].

Bien qu'il n'y ait pas de certitude sur le mécanisme en cause, il existe bien une augmentation de fréquence des hyperhaploïdies avec un risque de transmission mais celuici reste faible puisque plus de 90 à $95 \%$ des spermatozoïdes retrouvés chez ces patients sont normaux. Néanmoins, le risque n'étant pas nul, l'intérêt du recours à une procédure de diagnostic préimplantatoire mérite d'être discuté.

\section{CRITERES PRONOSTIQUES DE CONSERVATION DE LA SPERMATOGENÈSE}

Une seule étude [24] a cherché à définir des facteurs permettant de prédire la présence de spermatozoïdes lors de la biopsie testiculaire spécifiquement dans le syndrome de Klinefelter. Sur une série de 20 patients, l'extraction de spermatozoïdes a été positive pour 9 patients $(45 \%)$, négative pour les 11 autres. Les valeurs de FSH et d'Inhibine B ne sont pas différentes dans les 2 groupes. Le volume testiculaire, la testostéronémie basale et sa réponse lors du test à l'hCG sont significativement plus élevées dans le groupe ayant des spermatozoïdes. Il est toutefois impossible de définir, pour quelque critère que ce soit, une valeur seuil utilisable à l'échelon individuel pour prédire la présence de spermatozoïdes. En effet, dans d'autres études, des biopsies ont été positives alors que le volume testiculaire était inférieur à $3 \mathrm{ml}[14,27]$. S'il est intéressant de noter la différence dans i'intensité de l'altération leydigienne satellite du trouble de la spermatogenèse dans les deux groupes, avec et sans spermatozoïdes, le chevauchement des valeurs ne permet pas non plus de prédiction formelle à l'échelon individuel.

\section{CONCLUSION}

Les méthodes de microinjection intraovocytaire ont pu être appliquées à des patients présentant un syndrome de Klinefelter. Les publications font état de résultats positifs aussi bien à partir de spermatozoïdes éjaculés : 5 enfants nés pour 9 cas de syndrome de Klinefelter avec oligospermie extrême ; soit à partir de spermatozoïdes testiculaires : 32 enfants nés pour 93 cas de syndrome de Klinefelter. Bien que l'on puisse tout à fait suspecter un biais de publication, les échecs n'ayant probablement pas fait l'objet de publication, ces résultats sont tout de même inattendus si on se réfère au pronostic traditionnellement admis dans le syndrome de Klinefelter. Le taux de spermatozoïdes aneuploïdes est inférieur à celui que l'on pouvait craindre puisque 90 à $95 \%$ des spermatozoïdes ont une formule chromosomique normale. Les aneuploïdies gonosomiques ne paraissent pas très fréquentes puisqu'un seul fotus 47,XXY a été rapporté parmi les enfants ou fotus des grossesses évolutives. Le risque d'aneuplö̈die gonosomique fait tout de même discuter l'intérêt d'une procédure de diagnostic préimplantatoire dans ce contexte. Les données de la littérature ne permettent pas encore clairement de connaître la proportion réelle des cas de syndrome de Klinefelter pour lesquels on peut disposer de spermatozoïdes ayant un bon potentiel de développement embryonnaire. Les moyens permettant de repérer ces patients restent aussi à inventorier.

\section{REFERENCES}

1. BERGERE M., WAINER R., NATAF V., et al. : Biopsied testis cells of four 47,XXY patients: fluorescence in situ hybridization and ICSI results. Hum. Reprod., 2002, $17: 32-37$.

2. BIELANSKA M., TAN S.L.. AO A. : Fluorescence in situ hybridization of sex chromosomes in spermatozoa and spare preimplantation embryos of a Klinefelter $46, \mathrm{XY} / 47, \mathrm{XXY}$ male. Hum. Reprod.. 2000, $15: 440-444$.

3. BOURNE H., RICHINGS N., HARARI O., et al. : The use of intracytoplasmic sperm injection for the treatment of severe and extreme male infertility. Reprod. Fertil. Dev., 1995, 7 : 237-245.

4. BOURNE H., STERN K., CLARKE G., PERTILE M., SPEIRS A., BAKER H.W. : Delivery of normal twins following the intracytoplasmic injection of spermatozoa from a patient with 47, XXY Klinefelter's syndrome. Hum. Reprod., 1997, 12 : 2447-2450.

5. CHEVRET E., ROUSSEAUX S., MONTEIL M., et al. : Increased incidence of hyperhaploid $24, X Y$ spermatozoa detected by three- colour FISH in a $46, X Y / 47, X X Y$ male. Hum. Genet., 1996, $97: 171-175$.

6. COZZI J., CHEVRET E., ROUSSEAUX S., et al. : Achievement of meiosis in XXY germ cells : study of 543 sperm karyotypes from an XY/XXY mosaic patient. Hum. Genet., 1994, 93 : 32 34.

7. CRUGER D., TOFT B., AGERHOLM I., FEDDER J., HALD F., BRUUN-PETERSEN G. : Birth of a healthy girl after ICSI with ejaculated spermatozoa from a man with non-mosaic Klinefelter's syndrome. Hum. Reprod., 2001, 16 : 1909-1911.

8. DEVROEY P., LIU J., NAGY Z., et al. : Pregnancies after testicular sperm extraction and intracytoplasmic sperm injection in non-obstructive azoospermia. Hum. Reprod., 1995, 10 : 14571460 .

9. ESTOP A.M., MUNNE S., CIEPLY K.M., VANDERMARK K.K., LAMB A.N., FISCH H. : Meiotic products of a Klinefelter $47, \mathrm{XXY}$ male as determined by sperm fluorescence in situ hybridization analysis. Hum. Reprod., 1998, 13 : 124-127.

10. Finkelstein S., MUKAMEL E., YAVETZ H., PAZ G., AVIVI L. : Increased rate of nondisjunction in sex cells derived from low-quality semen. Hum. Genet., 1998, 102 : 129-137. 
11. FORESTA C., GALEAZZI C., BETTELLA A., STELLA M., SCANDELLARI C. : High incidence of sperm sex chromosomes aneuploidies in two patients with Klinefelter's syndrome. J. Clin. Endocrinol. Metab., 1998, 83 : 203-205.

12. FORESTA C., GALEAZZI C., BETTELla A., et al. : Analysis of meiosis in intratesticular germ cells from subjects affected by classic Klinefelter's syndrome. J. Clin. Endocrinol. Metab., $1999,84: 3807-3810$.

13. FRIBERG J., KJESSLER B. : Sperm-agglutinating antibodies and testicular morphology in fifty-nine men with azoospermia or cryptozoospermia. Am. J. Obstet. Gynecol., 1975, 121 : 987-990.

14. FRIEDLER S., RAZIEL A., STRASSBURGER D., SCHACHTER M., BERN O., RON-EL R. : Outcome of ICSI using fresh and cryopreserved-thawed testicular spermatozoa in patients with non-mosaic Klinefelter's syndrome. Hum. Reprod., 2001, $16: 2616-2620$.

15. GUICHAOUA M.R., DELAFONTAINE D., NOE L.B., LUCIANI J.M. : L'infertilité masculine d'origine chromosomique. Contracept. Fertil. Sex., 1993, 21 : 113-121.

16. GUTTENBACH M., MICHELMANN H.W., HINNEY B., ENGEL W., SCHMID M. : Segregation of sex chromosomes into sperm nuclei in a man with $47, \mathrm{XXY}$ Klinefelter's karyotype: a FISH analysis. Hum. Genet., 1997, $99: 474-477$.

17. HARARI O., BOURNE H., BAKER G., GRONOW M., JOHNSTON I. : High fertilization rate with intracytoplasmic sperm injection in mosaic Klinefelter's syndrome. Fertil. Steril., 1995, $63: 182-184$.

18. HINNEY B., GUTTENBACH M., SCHMID M., ENGEL W., MICHELMANN H.W. : Pregnancy after intracytoplasmic sperm injection with sperm from a man with a 47, XXY Klinefelter's karyotype. Fertil. Steril., 1997, $68: 718-720$.

19. KITAMURA M., MATSUMIYA K., KOGA M., et al. : Ejaculated spermatozoa in patients with non-mosaic Klinefelter's syndrome. Int. J. Urol., 2000, $7: 88-92$.

20. KYONO K., FUKUNAGA N., HAIGO K., CHIBA S., ARAKI Y. : Pregnancy achieved following ICSI from a man with Klinefelter's syndrome and spinal cord injury. Hum. Reprod., 2001, $16: 2347-2349$.

21. LARON Z., DICKERMAN Z., ZAMIR R., GALATZER A. : Paternity in Klinefelter's syndrome - a case report. Arch. Androl., 1982, $8: 149-151$.

22. LEVRON J., AVIRAM-GOLDRING A., MADGAR I., RAVIV G., BARKAI G., DOR J. : Sperm chromosome analysis and outcome of IVF in patients with non-mosaic Klinefelter's syndrome. Fertil. Steril., 2000, 74 : 925-929.

23. LUCIANI J.M., MATTEI A., DEVICTOR-VUILLET M., RUBIN, STAHL A., VAGUE J. : Etude des chromosomes méiotiques dans un cas de maladie de Klinefelter avec spermatogenèse et caryoype 46,XY-47,XXY. Ann. Genet., 1970, 13 : 249-253.

24. MADGAR I., DOR J., WEISSENBERG R., RAVIV G., MENASHE Y., LEVRON J. : Prognostic value of the clinical and laboratory evaluation in patients with non-mosaic Klinefelter syndrome who are receiving assisted reproductive therapy. Fertil. Steril., 2002, 77 : 1167-1169.

25. MARTINI E., GERAEDTS J.P., LIEBAERS I., et al. : Constitu- tion of semen samples from XYY and XXY males as analysed by in situ hybridization. Hum. Reprod., 1996, 11 : 1638-1643.

26. NIELSEN J., WOHLERT M. : Chromosome abnormalities found among 34,910 newborn children: results from a 13-year incidence study in Arhus, Denmark. Hum. Genet., 1991, 87 : 8183.

27. NODAR F., DE VINCENTIIS S., OLMEDO S.B., PAPIER S., URRUTIA F., ACOSTA A.A. : Birth of twin males with normal karyotype after intracytoplasmic sperm injection with use of testicular spermatozoa from a non-mosaic patient with Klinefelter's syndrome. Fertil. Steril., 1999, 71 : 1149-1152.

28. PALERMO G.D., SCHLEGEL P.N., SILLS E.S., et al. : Births after intracytoplasmic injection of sperm obtained by testicular extraction from men with non-mosaic Klinefelter's syndrome. N. Engl. J. Med., 1998, 338 : 588-590.

29. PALERMO G.D., SCHLEGEL P.N., HARIPRASHAD J.J., et al. : Fertilization and pregnancy outcome with intracytoplasmic sperm injection for azoospermic men. Hum. Reprod., 1999, 14 : $741-748$.

30. PAULSEN C., GORDON D., CARPENTER R., GANDY H., DRUCKER W. : Klinefelter's syndrome and its variants: a hormonal and chromosomal study. Recent. Prog. Horm. Res., 1968, $24: 321-363$.

31. POULAKIS V., WITZSCH U., DIEHL W., DE VRIES R., BECHT E., TROTNOW S. : Birth of two infants with normal karyotype after intracytoplasmic injection of sperm obtained by testicular extraction from two men with non-mosaic Klinefelter's syndrome. Fertil. Steril., 2001, $76: 1060-1062$.

32. REUBINOFF B.E., ABELIOVICH D., WERNER M., SCHENKER J.G., SAFRAN A., LEWIN A. : A birth in non-mosaic Klinefelter's syndrome after testicular fine needle aspiration, intracytoplasmic sperm injection and preimplantation genetic diagnosis. Hum. Reprod., 1998, 13 : 1887-1892.

33. RON-EL R., FRIEDLER S., STRASSBURGER D., KOMAROVSKY D., SCHACHTER M., RAZIEL A. : Birth of a healthy neonate following the intracytoplasmic injection of testicular spermatozoa from a patient with Klinefelter's syndrome. Hum. Reprod., 1999, $14:$ 368-370.

34. RON-EL R., RAZIEL A., STRASSBURGER D., SCHACHTER M., BERN O., FRIEDLER S. : Birth of healthy male twins after intracytoplasmic sperm injection of frozen-thawed testicular spermatozoa from a patient with nonmosaic Klinefelter syndrome. Fertil. Steril., 2000, $74: 832-833$.

35. RON-EL R., STRASSBURGER D., GELMAN-KOHAN S., FRIEDLER S., RAZIEL A., APPELMAN Z. : A 47,XXY fetus conceived after ICSI of spermatozoa from a patient with nonmosaic Klinefelter's syndrome: case report. Hum. Reprod., 2000, $15: 1804-1806$.

36. SKAKKEBAEK N.E. : Two types of tubules containing only Sertoli cells in adults with Klinefelter's syndrome. Nature, 1969, $223: 643-645$.

37. STAESSEN C., COONEN E., VAN ASSCHE E., et al. : Preimplantation diagnosis for $\mathrm{X}$ and $\mathrm{Y}$ normality in embryos from three Klinefelter patients. Hum. Reprod., 1996, $11: 1650-1653$.

38. TERZOlI G., LALATTA F., LOBBIANI A., SIMONI G., COLUCCI G. : Fertility in a $47, \mathrm{XXY}$ patient: assessment of bio- 
logical paternity by deoxyribonucleic acid fingerprinting. Fertil. Steril., 1992, $58: 821-822$.

39. TOURNAYE H., CAMUS M., GOOSSENS A., et al. : Recent concepts in the management of infertility because of non-obstructive azoospermia. Hum. Reprod., 1995, 10: 115-119.

40. TOURNAYE H., LIU J., NAGY P.Z., et al. : Correlation between testicular histology and outcome after intracytoplasmic sperm injection using testicular spermatozoa. Hum. Reprod., $1996,11: 127-132$.

41. TOURNAYE H., STAESSEN C, LIEBAERS I., et al. : Testicular sperm recovery in nine $47, \mathrm{XXY}$ Klinefelter patients. Hum. Reprod,, 1996, 11 : 1644-1649.

42. TOURNAYE H., CAMUS M., VANDERVORST M., et al. : Surgical sperm retrieval for intracytoplasmic sperm injection. Int. J. Androl., 1997, $20: 69-73$.

43. YAMAMOTO Y., SOFIKITIS N., MIO Y., LOUTRADIS D., KAPONIS A., MIYAGAWA I. : Morphometric and cytogenetic characteristics of testicular germ cells and Sertoli cell secretory function in men with non-mosaic Klinefelter's syndrome. Hum. Reprod., 2002, 17 : 886-896.

\begin{abstract}
Klinefelter's syndrome and intracytoplasmic sperm injection (review)
\end{abstract}

\section{Gerald RAVEROT, Hervé LEJEUNE}

Historically, Klinefelter's is the typical clinical form of secretory azoospermia with no hope of achieving biological paternity. However, ICSI, either from ejaculated sperm or following testicular sperm extraction, have been recently applied to patients with Klinefelter's syndrome. Papers published until June 2002 have reported microinjections with ejaculated sperm in 9 cases of Klinefelter's syndrome with extreme oligospermia with the following overall results: 79 mature oocytes, 52 fertilized oocytes, 31 embryos, 18 transferred embryos, 6 pregnancies, 5 births, or from testicular spermatozoa, in 93 cases, with the following results: 347 oocytes, 193 fertilized oocytes, 149 embryos, 78 embryos transferred out of 37 cycles, 24 clinical pregnancies and 2 positive pregnancy tests, and 32 births. Although a publication bias was very likely (successful attempts were published, failed attempts were probably not published), these results were unexpected based on the traditional view on Klinefelter's syndrome. The rate of aneuploid spermatozoa also appeared to be lower than expected. The real proportion of Klinefelter patients in whom spermatozoa with a good potential for embryonic development can be retrieved and the means of identifying these patients remain to be established.

Key-Words: Klinefelter, azoospermia, testicular, sperm, extraction, ICSI, biopsy 